\title{
Quantitative Determination of Thermal Fields and Transformation Rates in Rapidly Solidifying Aluminum by Numerical Modeling and In-situ TEM
}

\author{
Can Liu ${ }^{1}$, Kai Zweiacker ${ }^{1}$, Joseph T. McKeown ${ }^{2}$, Thomas LaGrange ${ }^{3}$, Bryan W. Reed ${ }^{3}$, Geoffrey H. \\ Campbell $^{2}$ and Jörg M.K. Wiezorek ${ }^{1}$ \\ 1. Department of Mechanical Engineering and Materials Science, University of Pittsburgh, \\ Pittsburgh, PA 15261 \\ 2. Materials Science Division, Lawrence Livermore National Laboratory, Livermore, CA 94551 \\ 3. Integrated Dynamic Electron Solutions, Inc, Pleasanton, CA 94566
}

The dynamic transmission microscope (DTEM) offers incomparable nano-scale spatiotemporal resolution that is advantageous for characterizing irreversible transient processes in various material systems [1]. Previously, we utilized bright field imaging and diffraction in DTEM with nano-second temporal resolution to characterize the dynamics of rapid solidification (RS) in pure $\mathrm{Al}$ and $\mathrm{Al}-\mathrm{Cu}$ alloy thin films in single-shot, single-image acquisition mode [2-4]. Recent developments in DTEM instrument enable a single-shot, multiple-image acquisition mode (Movie-mode) [5], which significantly reduces potential uncertainty and experimental error when measuring parameters such as interface velocity during rapid solidification process. However, it is inherently challenging to measure the temperature evolution during the RS transformation cycle while performing in-situ DTEM observations. Knowledge of the thermal field evolution in correlation to the solidification front velocities would greatly benefit quantitative understanding of RS process in material systems.

Here we report results of enthalpy-based numerical modeling performed in a COMSOL ${ }^{\circledR}$ multiphysics environment that have been validated by experimental observations to quantitatively determine the thermal field evolution during RS. Figure 1 represents a schematic illustration of a possible timing sequence for Movie-mode DTEM experimentation and an example of an image series obtained for pulsed-laser induced melting and subsequent re-solidification in 80-nm-thick pure $\mathrm{Al}$ thin film. The image series includes nine images spanning from $0 \mu \mathrm{s}$ to $20.4 \mu \mathrm{s}$ after the melting laser pulse, covering onset of melting to completion of solidification using a 50-ns electron pulse duration and a $2.5-\mu$ s interframe time spacing. Several image sequences were used to calculate the evolution of the melt-pool size (radius, R) and interface velocity during RS. Figure 2 shows the thermal field distribution predicted by simulations during RS and the comparison between the experimentally determined time evolution of the melt pool area and solidification front velocity from the time-resolved image sequences and those computed by simulations. The details of the thermal field and associated velocity evolution behavior will be discussed.

References

[1] King, W.E., et al., Journal of Applied Physics (2005) 97: 111101.

[2] Kulovits, A.K., et al., Philosophical Magazine Letters (2011) 91: 287.

[3] McKeown, J.T., et al., Microscopy and Microanalysis (2012) Vol. 18 (Suppl. 2): 602.

[4] McKeown, J.T., et al., Acta Materialia (2014) 65: 56.

[5] LaGrange T., et al., Micron (2012) 43: 1108.

[6] Work was performed under the auspices of the U.S. Department of Energy, Office of Basic Energy Sciences, Division of Materials Sciences and Engineering for FWP SCW0974 by Lawrence Livermore National Laboratory under Contract DE-AC52-07NA27344.The research activities at the 
University of Pittsburgh received support from the National Science Foundation, Division of Materials Research, Metals \& Metallic Nanostructures program through Grant No. DMR 1105757.

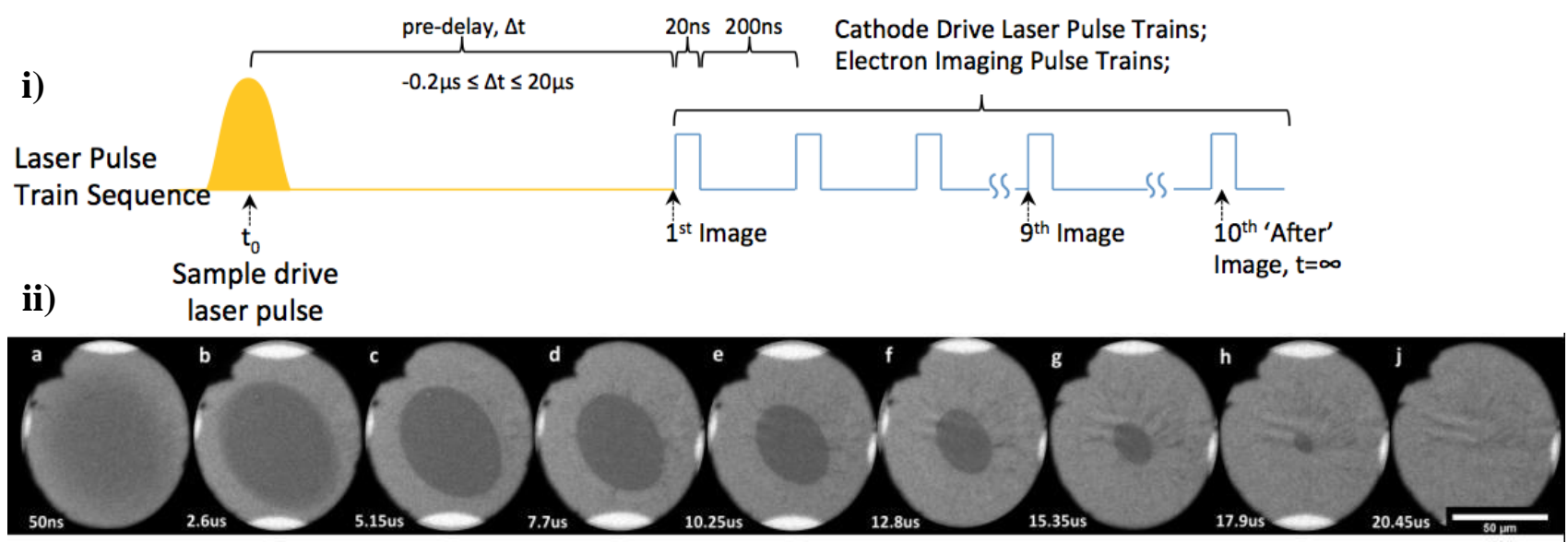

Figure 1. i) Schematic illustration of Movie-mode DTEM with selected pre-delay time and interframe time spacing and ii) an example of image sequence showing laser-induced melting and $\mathrm{RS}$ in Al thin films recorded by in-situ Movie-mode DTEM using a 50-ns electron pulse duration and a $2.5-\mu$ s interframe time spacing

i)

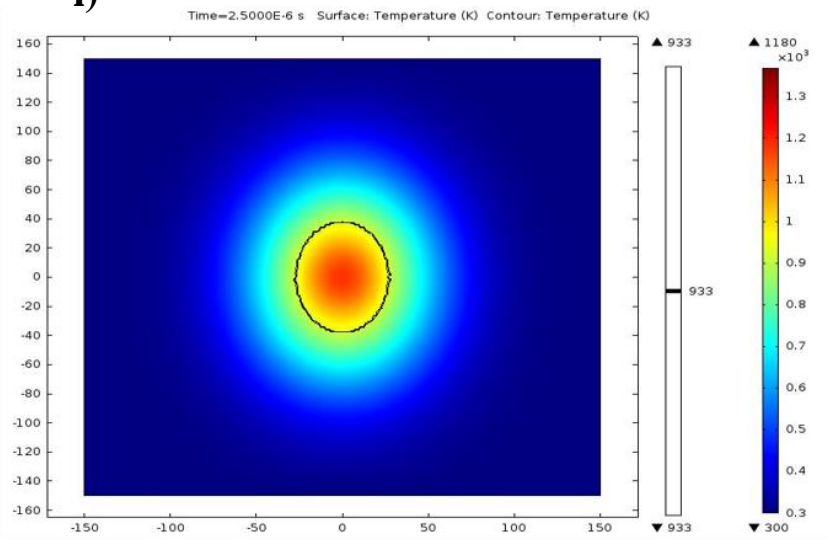

iii)

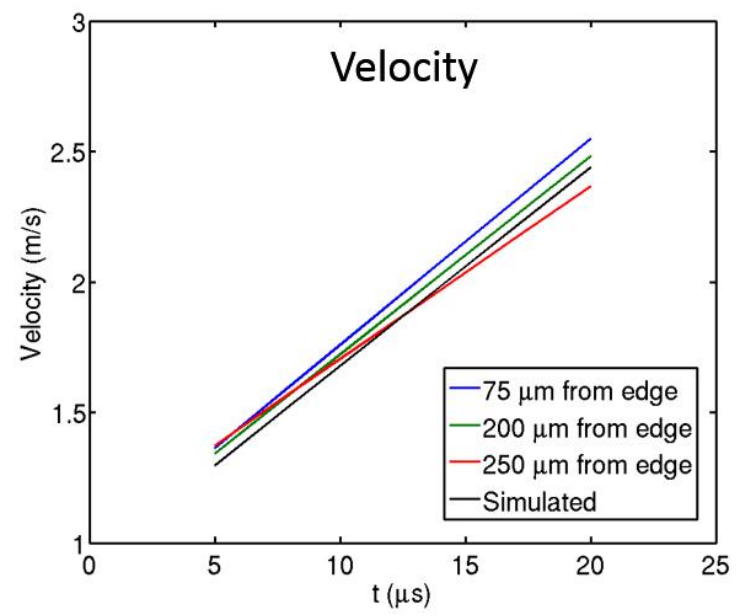

ii)

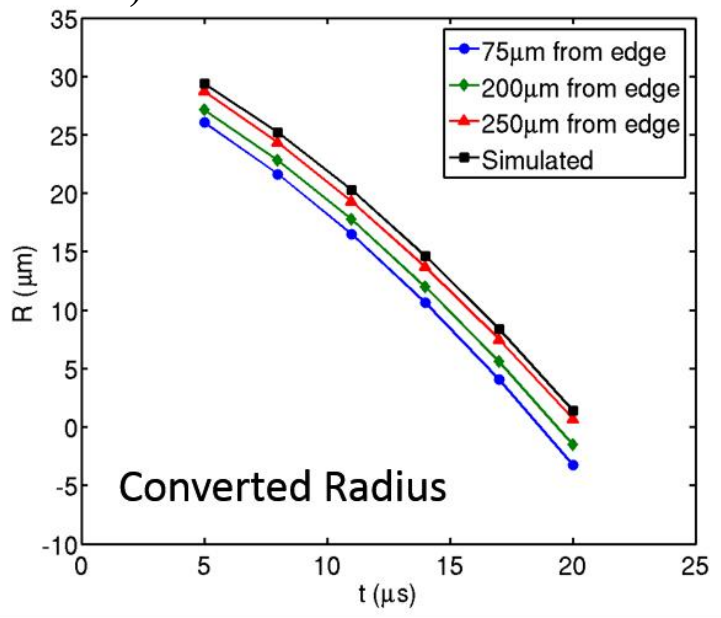

Figure 2. i) Temperature distribution in the pure $\mathrm{Al}$ film simulated by $\mathrm{COMSOL}^{\circledR}$ multi-physics model, ii) comparison between the experimentally determined converted radius evolution of the melt pool and simulated radius evolution and iii) simulated solidification velocity compared with velocity calculated from experimental observations 